\title{
Reduced Encoding of Diffusion Spectrum Imaging with Cross-term Correction
}

\author{
C. P. Lin ${ }^{1}$, W. Y. I. Tseng ${ }^{2}$, J. C. Weng ${ }^{1}$, V. J. Wedeen ${ }^{3}$, J. H. Chen ${ }^{1}$ \\ 1 Department of Electrical Engineering, National Taiwan University, Taipei, Taiwan \\ 2 Center for Optoelectronic Biomedicine, National Taiwan University Medical College, Taipei, Taiwan \\ 3 Department of Radiology, NMR Center, Massachusetts General Hospital
}

\begin{abstract}
Diffusion Spectrum MRI (DSI) has been demonstrated to be capable of defining orientations of intersecting fibers accurately. However, the scanning procedure is overly timeconsuming under the current sampling scheme. In this study, we developed a reduced encoding scheme of DSI and proved that decreasing the acquisition points to $62 \%$ is feasible by diffusion cross-term correction. We calculated the diffusion cross-term from the acquired data in the center of $q$ space by assuming that image corresponding to any diffusion encoding direction suffers $b$ values from diffusion gradients, imaging gradients and the cross-term between diffusion gradients and imaging gradients. The data in outer $q$ space was then corrected with diffusion cross-term and filled by the data acquired with opposite diffusion gradients according to the geometric symmetry of diffusion anisotropy. Both ex-vivo crossing capillaries phantom and ex-vivo rat brain models showed that this method can decrease DSI acquisition time while preserving the patterns and orientations of probability density function.
\end{abstract}

Keywords - diffusion MRI, DSI, cross-term

\section{INTRODUCTION}

Water molecule displacement restricted in an organized material reflects geometrical properties of the underlying microenvironment. Based on this tenet, Diffusion Spectrum MRI (DSI) has been exploited to reflect the distribution of fiber orientations by directly mapping of 3D probability density function (PDF) of proton diffusion through $\mathbf{q}$ space diffusion MRI technique [1]. Under the current sampling scheme, our previous reports show that DSI has accuracy close to noise limit [2] and can be capable of resolving complex fiber architecture in cortical gray matter [3].

Currently, DSI scanning requires 515 diffusion-encoding directions, it is very time consuming. In this study, by our newly proposed method, we are able to reconstruct 515 diffusion-encoding directions successfully from 319 directions (by reducing encodings in $\mathbf{q}$ space) according to the geometric symmetry of diffusion anisotropy and diffusion cross-term correction. We calculated the diffusion cross-term from the acquired data in the center of $q$ space by assuming that image corresponding to any diffusion encoding direction suffers $b$ values from diffusion gradients, imaging gradients and the cross-term between diffusion gradients and imaging gradients [4]. Images acquired with opposite diffusion gradients will suffer the same $b$ values from diffusion gradients and imaging gradients but negative on diffusion cross-terms [5]. The data in outer $q$ space was then corrected with diffusion cross-term and filled by the data acquired with opposite diffusion gradients according to the geometric symmetry of diffusion anisotropy. The experimental results of phantom and rat brain models both showed that this method is useful in the reduction of DSI acquisition time to $62 \%$ while preserving the PDF patterns and orientations.

\section{METHODOLOGY}

Adult Wistar rats were euthanized under deep general anesthesia with intra-peritoneal injection of sodium pentobarbital, $100 \mathrm{mg} / \mathrm{kg}$. After being exsanguinated, the rats were perfused transcardially with physiological saline followed by $4 \%$ formaldehyde solution. Rat brains were dissected from the cranium and were placed fixedly in an acrylic holder filled with $4 \%$ formaldehyde solution. MR experiment was performed in a $3 T$ MRI Biospect system (Bruker, Germany). DSI images were acquired using spin echo diffusion sequence with 319 diffusion-encoding directions. These directions comprised of half isotropic 3D grid points in the $\mathbf{q}$ space contained within a spherical volume of 5 radial increments with a spherical volume of 3 radial increments on the opposite side of $\mathbf{q}$ space. The spatial modulation is $\mathbf{q}=\gamma \mathbf{g} \delta$ where $\gamma$ is the proton gyromagnetic ratio, $\mathbf{g}$ is the diffusion-encoding gradient, and $\delta$ is the time duration of the diffusion gradient. The maximum diffusion gradient intensity $=480 \mathrm{mTm}^{-1}, \delta=10$ $\mathrm{ms}$ and diffusion time $=20 \mathrm{~ms}$, and the maximum diffusion sensitivity $b_{\max }=27,500 \mathrm{~s} \mathrm{~mm}^{-2}$. Using FOV of $2 \mathrm{~cm}$, matrix size of $128 \times 128$, slice thickness of $1 \mathrm{~mm}$, we then obtained DSI images at $160-\mu \mathrm{m}$ in-plane resolution.

Phantom comprised of sheets of parallel plastic capillaries with inner and outer diameters of $50 \mu \mathrm{m}$ and $350 \mu \mathrm{m}$, respectively. The capillaries were filled with water and sheets of two different orientations. These two orientations were stacked on each other in an interleaved fashion [2]. DSI was obtained from the phantoms by using stimulated echo diffusion sequences with the same encoding strategy used in the rat model. Images were acquired at $0.7-\mathrm{mm}$ inplane resolution and $3.5 \mathrm{~mm}$ thickness. Maximum diffusion gradient had intensity $=112 \mathrm{mTm}^{-1}$, duration $=6 \mathrm{~ms}$ and diffusion time $=250 \mathrm{~ms}$, which yielded maximum diffusion sensitivity $=8,000 \mathrm{~s} \mathrm{~mm}^{-2}$.

DSI images within the spherical volume of radius 3 were used to calculate the diffusion cross-term. We assume that image corresponding to any diffusion encoding direction suffers $b$ values from diffusion gradients, image gradients and the cross-term between diffusion gradients and image 
gradients. For example, the echo signal at $(1, m, n)$ in $\mathbf{q}$ space can be expressed by

$$
\begin{gathered}
S_{(1, m, n)} / S_{0}=\exp \left[-\left(b_{D}+b_{1}+l b_{x x}+m b_{y y}+n b_{z z}+l b_{x y}+l b_{x z}+\right.\right. \\
\left.\left.m b_{y x}+m b_{y z}+n b_{z x}+n b_{z y}\right) D_{l m n}\right]
\end{gathered}
$$

, where $b_{D}$ is the $b$ value from diffusion gradients, $b_{I}$ is the $b$ value from image gradients and $b_{\alpha \beta}$ is the cross-term $b$ value contributed from diffusion gradient in $\alpha$ direction and image gradients in $\beta$ direction, respectively. $D_{\operatorname{lmn}}$ is the apparent diffusion coefficient in the direction of $(1, \mathrm{~m}, \mathrm{n})$ and can be calculated by

$$
D_{1 m n}=-\log \left(S_{(1, m, n)} S_{(-1,-m,-n)} / S^{2}{ }_{(0,0,0)}\right) / 2 b_{D}
$$

Using the fact that

$$
\begin{array}{r}
\log \left(S_{(1, m, n)} / S_{(-1,-m,-n)}\right)=2 \\
2\left(b_{x x}+m b_{y y}+n b_{z z}+1 b_{x y}+l b_{x z}+\right. \\
\\
\left.m b_{y x}+m b_{y z}+n b_{z x}+n b_{z y}\right) D_{l m n}
\end{array}
$$

, we can then obtain the cross-term b-values by the echo signal encoded within the sphere of radius 3 .

DSI data were then filled to 515 points in $q$ space from the diffusion cross-term corrected echo signal, which was originally acquired with opposite diffusion gradients, i.e.

$S_{(-i,-j,-k)}=S_{(i, j, k)} \exp \left[2\left(i b_{x x}+j b_{y y}+k b_{z z}+i b_{x y}+i b_{x z}+j b_{y x}+j b_{y z}\right.\right.$

$$
\left.\left.+\mathrm{kb}_{\mathrm{zx}}+\mathrm{kb}_{\mathrm{zy}}\right) \mathrm{D}_{\mathrm{ijk}}\right] \text {. }
$$

The reconstruction of DSI data, $S(\mathbf{q})$, was based on the relationship that echo signal $\mathrm{S}(\mathbf{q})$ and diffusion PDF $P(\mathbf{r})$ was a Fourier pair, namely, $\mathrm{S}(\mathbf{q})=\mathrm{F}[P(\mathbf{r})]$ [6]. The maximum PDF orientation was then calculated and normalized for all voxels.
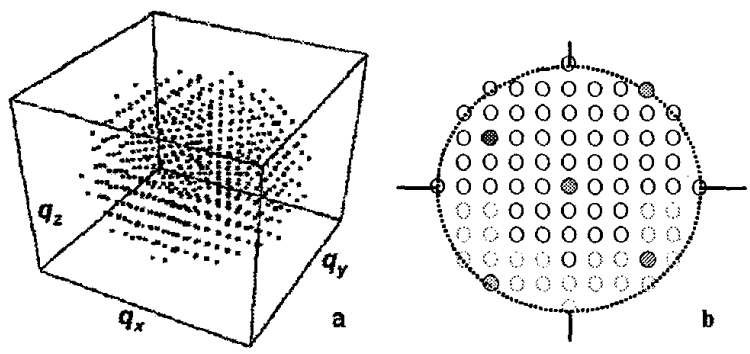

Fig. 1. Encoding scheme of DSI and cross-term corrected reduced encoding of DSI. The DSI diffusion-encoding directions comprised of isotropic 3D grid points in the $\mathbf{q}$ space contained within a spherical volume of 5 radial increments (a). Figure 1-b shows the coronal view of our reduced encoding method, where " $\mathrm{o}$ " is the encoding directions of the reduced encoding of DSI in q space. The semiopaque "o" points in q space are calculated from the points with opposite diffusion gradients after cross-term correction. For example, the semiopaque green and red points in $\mathbf{q}$ space are calculated from the green and red points, respectively.

\section{RESULTS}

DSI of phantoms clearly shows that the cross-term corrected reduced encoding of DSI can preserve the original PDF patterns and orientations (Fig. 2- a, b). The deviation angle between these two figures is $-0.4^{\circ} \pm 3.87^{\circ}$.

Fig. 3 shows the DSI of rat hippocampus with and without cross-term correction. The deviation angle of the maximum PDF orientation between these two figures for the voxel with PDF length $>0.3$ is $1.59^{\circ} \pm 7.1^{\circ}$. Our result show that the complex fiber structure of hippocampus can still be resolved under the cross-term corrected reduced encoding of DSI [3].

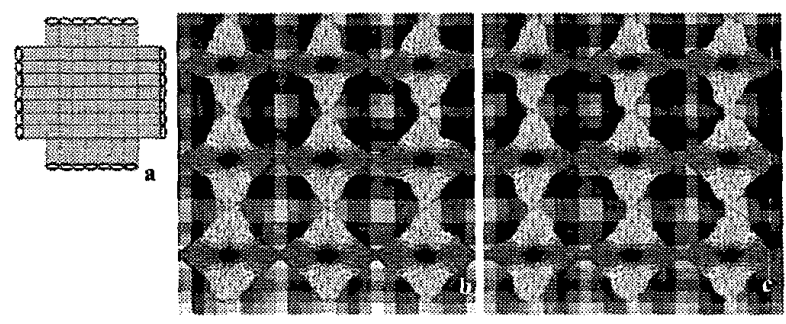

Fig. 2. $90^{\circ}$ phantom (a) with cross-term corrected reduced encoding of DSI in read direction (b) and DSI with 515 encoding directions (c). The deviation angle between Fig2-b and Fig $2-\mathrm{c}$ is $-0.4^{\circ}=3.87^{\circ}$. This result shows that cross-term corrected reduced encoding can be used to decrease the data acquisition time while preserving the fiber orientations.

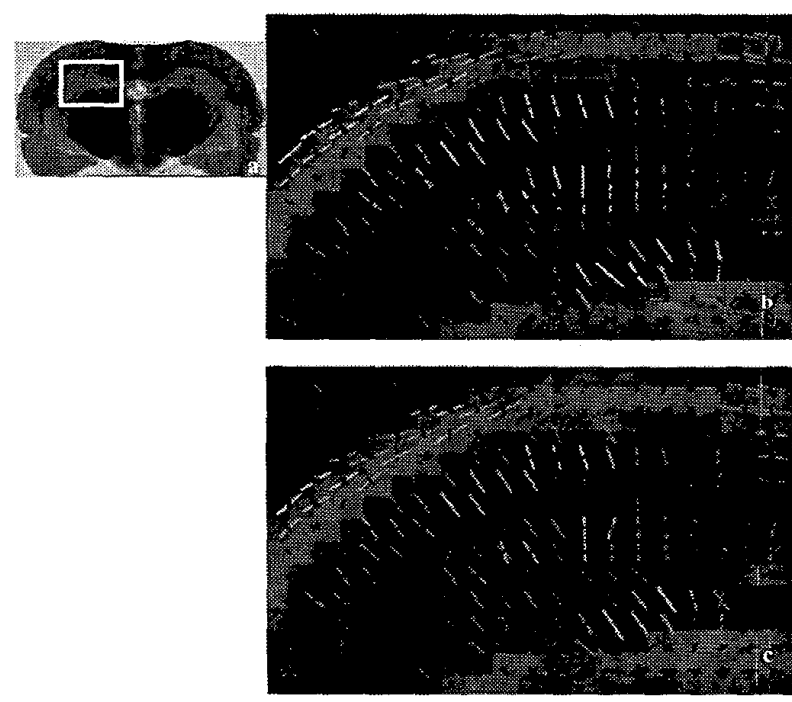

Fig. 3. T2WI (a) and DSI of rat hippocampus with original 515 diffusion encoding directions (b) and cross-term corrected reduced encoding (c). The complex fiber structure of hippocampus can also be resolved under the cross-term corrected reduced encoding of DSI.

\section{Discussion}

The reduced-encoding method of getting DSI can be applied on either read, phase or slice direction. DSI result of 
phantom clearly shows that the PDF is unstable in either direction of under-sampling of DSI (Fig. 4- a,b). Our results show that this unstable can be removed by diffusion crossterm correction.

The deviation angle of $-0.4^{\circ} \pm 3.87^{\circ}$ for phantom and $1.59^{\circ}$ $\pm 7.1^{\circ}$ for rat brain may come from the decrease of image numbers and error of estimating cross-term with lower $b$ values. This error can be improved by $b$ matrix calculation directly from MR sequence [4]. Error of $1.59^{\circ} \pm 7.1^{\circ}$ for rat brain can be decrease to $0.74^{\circ} \pm 4.84^{\circ}$ for the voxel with maximum PDF length longer than 0.5. This result shows that the fibers with high diffusion anisotropy can still be resolved under our cross-term corrected reduced encoding method.
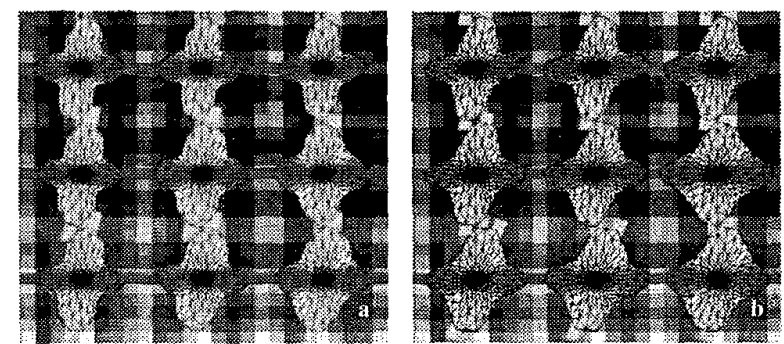

Fig. 4. DSI of $90^{\circ}$ phantom with reduced encoding in read direction (a) and reduced encoding in phase direction (b). Our results show that the reduced encoding of DSI without cross-term correction will lead unstable orientations.

\section{CONCLUSION}

Our results show that the diffusion cross-term can be calculated from the image data acquired with lower $b$ values. The data in outer $\mathbf{q}$ space can be calculated by cross-term corrected data that acquired with opposite diffusion gradients. The error of our method is close to the noise limit for the fibers with higher diffusion anisotropy. In conclusion, using cross-term corrected reduced encoding of DSI, we have decreased the sampling time of DSI to $62 \%$ while preserving the patterns and orientations of probability density function

\section{ACKNOWLEDGMENT}

The authors are indebted to Dr. Keng-Chen Liang and Dr. Der-Yow Chen for their helpful advice to this study. This study is supported in part by the National Health Research Institutes grant NHRI-EX90-9018EP and the Program for Promoting Academic Excellence of Universities A-91-NFA01-2-4.

\section{REFERENCES}

[1] V. J. Wedeen, T. G. Reese, D. S. Tuch, M. R. Weigel, J.G. Dou, R. M. Weiskoff, and D. Chessler, "Mapping fiber orientation spectra in cerebral white matter with Fourier-transform diffusion MRI," presented at Proceedings of the 9th Annual Meeting of ISMRM, Denver, Colorado, USA, 2000.
[2] C. P. Lin, V. J. Wedeen, C. Yao, J. H. Chen, and W. Y. I. Tseng, "Validation of Diffusion Spectrum Magnetic Resonance Imaging with Registered Manganeseenhanced Optic Tracts and Phantom," presented at Proceedings of ISMRM diffusion workshop, Saint-Malo, France, 2002.

[3] W. Y. I. Tseng, C. P. Lin, J. H. Chen, and V. J. Wedeen, "Diffusion Spectrum Imaging of Complex Cortical Cytoarchitecture in Adult Rats," presented at Proceedings of ISMRM 10th Ann Meeting, Hawaii, USA, 2002.

[4] J. Mattiello, P. J. Basser, and D. Le Bihan, "b factor: analytical calculation of the $b$ matrix in diffusion imaging," in Diffusion and perfusion magnetic resonance imaging: applications to functional $M R I$, vol. Chapter 5: Part II, D. Le Bihan, Ed. New York: Raven Press, Ltd., 1995, pp. 77-90.

[5] M. Neeman, J. P. Freyer, and L. O. Sillerud, "A simple method for obtaining cross-term-free images for diffusion anisotropy studies in NMR microimaging," Magn Reson Med, vol. 21, pp. 138-43., 1991.

[6] P. T. Callaghan, Principles of nuclear magnetic resonance microscopy, 1 ed: Oxford: Clarendon Press, 1991. 